\title{
Phase II study of trastuzumab in combination with S-1 plus cisplatin in HER2-positive gastric cancer (HERBIS-1)
}

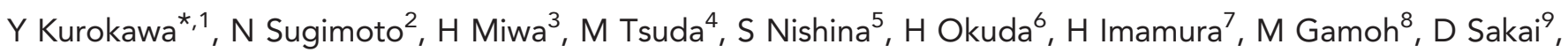
T Shimokawa $^{10}$, Y Komatsu $^{11}$, Y Doki ${ }^{1}$, T Tsujinaka ${ }^{12}$ and H Furukawa ${ }^{13}$

${ }^{1}$ Department of Gastroenterological Surgery, Osaka University Graduate School of Medicine, 2-2-E2, Yamadaoka, Suita 565-0871, Japan; ${ }^{2}$ Department of Clinical Oncology, Osaka Medical Center for Cancer and Cardiovascular Diseases, 3-3-1, Nakamichi, Osaka 537-8511, Japan; ${ }^{3}$ Department of Upper Gastroenterology, Hyogo College of Medicine, 1-1, Mukogawa-cho, Nishinomiya 663-8501, Japan; ${ }^{4}$ Department of Gastroenterological Oncology, Hyogo Cancer Center, 13-70, Kitaoji-cho, Akashi 673-8558, Japan; ${ }^{5}$ Department of Medical Oncology, Kinki University Faculty of Medicine, 377-2, Ohnohigashi, Osakasayama 589-8511, Japan; ${ }^{6}$ Deparment of Medical Oncology, Keiyukai Sapporo Hospital, 1-1-14, Hondori, Sapporo 003-0027, Japan; ${ }^{7}$ Deparment of Surgery, Sakai Municipal Hospital, 1-1-1, Yasui-cho, Sakai 590-0064, Japan; ${ }^{8}$ Deparment of Medical Oncology, Osaki Citizen Hospital, 2-3-10, Furukawasenjujicho, Osaki 989-6183, Japan; ${ }^{9}$ Deparment of Frontier Science for Cancer and Chemotherapy, Osaka University Graduate School of Medicine, 2-2, Yamadaoka, Osaka 565-0871, Japan; ${ }^{10}$ Graduate School of Medicine and Engineering, University of Yamanashi, 4-4-37, Takeda, Kofu 400-8510, Japan; ${ }^{11}$ Deparment of Cancer Center, Hokkaido University Hospital, North 15, West 7, Kita-ku, Sapporo 060-8638, Japan; ${ }^{12}$ Deparment of Surgery, Kaizuka City Hospital, 3-10-20, Hori, Kaizuka 597-0015, Japan and ${ }^{13}$ Deparment of Surgery, Kinki University Faculty of Medicine, 377-2, Ohnohigashi, Osakasayama 589-8511, Japan

Background: S-1, an oral fluoropyrimidine, plus cisplatin (SP) is a standard regimen for advanced gastric cancer (AGC) in East Asia. To date, no studies have evaluated the efficacy and safety of trastuzumab combined with SP in patients with human epidermal growth factor receptor type 2 (HER2)-positive AGC.

Methods: Patients with HER2-positive AGC received S-1 (80-120 mg per day) orally on days 1-14, cisplatin $\left(60 \mathrm{mg} \mathrm{m}^{-2}\right.$ ) intravenously on day 1 , and trastuzumab (course $1,8 \mathrm{mg} \mathrm{kg}^{-1}$; course 2 onward, $6 \mathrm{mg} \mathrm{kg}^{-1}$ ) intravenously on day 1 of a 21 -day cycle. The primary end point was response rate (RR); secondary end points included overall survival (OS), progression-free survival (PFS), time to treatment failure (TTF), and adverse events.

Results: A total of 56 patients were enrolled. In the full analysis set of 53 patients, the confirmed RR was $68 \%$ (95\% confidence interval $(\mathrm{Cl})=54-80 \%)$, and the disease control rate was $94 \%(95 \% \mathrm{Cl}=84-99 \%)$. Median OS, PFS, and TTF were estimated as 16.0, 7.8, and 5.7 months, respectively. Major grade 3 or 4 adverse events included neutropaenia (36\%), anorexia (23\%), and anaemia (15\%).

Conclusions: Trastuzumab in combination with SP showed promising antitumour activity and manageable toxic effects in patients with HER2-positive AGC.

*Correspondence: Dr Y Kurokawa; E-mail: ykurokawa@gesurg.med.osaka-u.ac.jp

Received 27 September 2013; revised 4 December 2013; accepted 2 January 2014; published online 28 January 2014 (c) 2014 Cancer Research UK. All rights reserved 0007-0920/14 
Gastric cancer is the second leading cause of cancer deaths worldwide (Ferlay et al, 2010). A global standard regimen for to treat advanced gastric cancer (AGC) has not been established (Macdonald et al, 2001; Cunningham et al, 2008). In Western countries, regimens containing a fluoropyrimidine (fluorouracil or an oral preparation) plus a platinum compound, and usually including docetaxel or epirubicin, have been most widely used. In East Asia, including Japan and Korea, a fluoropyrimidine plus a platinum compound has been used as standard therapy (Koizumi et al, 2008; Kang et al, 2009).

Recent studies have shed new light on the molecular mechanisms underlying the development and progression of gastric cancer. Trastuzumab is a monoclonal antibody targeting human epidermal growth factor receptor type 2 (HER2) with two antigenspecific sites that bind to the juxtamembrane portion of the extracellular domain of the HER2 receptor, thereby preventing activation of its intracellular tyrosine kinase (Hudis, 2007). The Trastuzumab for Gastric Cancer (ToGA) study, an international phase III trial comparing chemotherapy consisting of cisplatin plus capecitabine or fluorouracil $v s$ trastuzumab plus chemotherapy in patients with HER2-positive AGC, demonstrated a survival benefit with the addition of trastuzumab (Bang et al, 2010). Currently, both the US Food and Drug Administration and the European Medicines Agency approved trastuzumab for the treatment of patients with HER2-positive AGC, and trastuzumab in combination with cisplatin plus capecitabine or fluorouracil is a standard treatment for HER2-positive AGC in the West.

$\mathrm{S}-1$ is a fluoropyrimidine preparation combining tegafur, a prodrug of 5-fluorouracil (5-FU), gimeracil, and oteracil potassium in a molar ratio of $1: 0.4: 1$. Gimeracil is a dihydropyrimidine dehydrogenase inhibitor, allowing high concentrations of 5-FU to be maintained (Shirasaka et al, 1996; Diasio, 1999). Two phase II studies (Sakata et al, 1998; Koizumi et al, 2000) in patients with AGC showed response rates (RRs) exceeding 40\%. The S-1 Plus cisplatin versus S-1 In RCT In the treatment for Stomach cancer (SPIRITS) phase III trial established S-1 plus cisplatin (SP) as a standard first-line regimen for AGC in the East (Koizumi et al, 2008; Japanese Gastric Cancer Association, 2011). However, SP plus trastuzumab has not been evaluated in patients with HER2-positive AGC to date. We therefore conducted this phase II study to evaluate the efficacy and safety of SP plus trastuzumab in HER2-positive AGC.

\section{PATIENTS AND METHODS}

Patients. We enrolled patients with histologically proven unresectable or recurrent HER2-positive tumours in the stomach or gastroesophageal junction. Human epidermal growth factor receptor type 2 status of tumours was evaluated using immunohistochemistry (IHC) and fluorescence in situ hybridisation (FISH). In the IHC testing, HER2 tumour cell-membrane immunostaining was scored using a four-grade scale $(0 / 1+/ 2+$ $13+)$ according to scoring scheme (ToGA score): 0 , no staining or membranous reactivity in $<10 \%$ of tumour cells; $1+$, weak, barely perceptible membranous reactivity in $>10 \%$ of tumour cells; $2+$, complete or basolateral membranous reactivity either nonuniform or weak in $\geqslant 10 \%$ of cells; and $3+$, complete or basolateral membranous reactivity of strong intensity in $\geqslant 10 \%$ of tumour cells (Hofmann et al, 2008; Bang et al, 2010). FISH analyses for HER2 status were carried out according to the manufacturer's procedure. The total numbers of HER2 and chromosome 17 signals were counted in at least 20 tumour cell nuclei in two different areas. The case with HER2/chromosome 17 ratio of $\geqslant 2.0$ was defined as FISH positive. In this study, only patients with IHC $3+$, or IHC $2+$ and FISH positive were eligible. Patients were required to have measurable lesions according to the Response Evaluation Criteria in Solid Tumors (RECIST), version 1.1 (Eisenhauer et al, 2009). Eligibility criteria also included: age between 20 and 75 years; Eastern Cooperative Oncology Group performance status score of 0 or 1; leukocyte count between 3500 and $12000 \mathrm{~mm}^{-3}$, neutrophil count $\geqslant 2000 \mathrm{~mm}^{-3}$, hemoglobin $\geqslant 9.0 \mathrm{~g} \mathrm{dl}^{-1}$, platelet count $\geqslant 100000 \mathrm{~mm}^{-3}$, serum bilirubin $<1.5 \mathrm{mg} \mathrm{dl}^{-1}$, creatinine clearance $\geqslant 60 \mathrm{ml} \mathrm{min}^{-1}$ calculated using the Cockcroft-Gault formula, serum creatinine $\leqslant 1.2 \mathrm{mg} \mathrm{dl}^{-1}$, serum aspartate aminotransferase and alanine aminotransferase $<100 \mathrm{IUl}^{-1}$; and baseline left ventricular ejection fraction $\geqslant 50 \%$. Patients were excluded from the study if they could not maintain sufficient oral intake, have massive ascites or pleural effusions, or had received prior chemotherapy or radiotherapy within 6 months before enrollment. The study protocol was approved by the Osaka Gastrointestinal Cancer Chemotherapy Study Group (OGSG) Steering Committee and the institutional review boards of all participating hospitals. All patients provided written informed consent before enrollment. This study was registered with UMIN-CTR, UMIN000005739.

Treatment. Trastuzumab was commercially obtained in this study. Patients received cisplatin $\left(60 \mathrm{mg} \mathrm{m}^{-2}\right)$ plus trastuzumab (course $1,8 \mathrm{mg} \mathrm{kg}^{-1}$; course 2 onward, $6 \mathrm{mg} \mathrm{kg}^{-1}$ ) intravenously on day 1 and oral S-1 twice daily at a dose based on body surface area $\left(<1.25 \mathrm{~m}^{2}, 40 \mathrm{mg} ; \geqslant 1.25\right.$ to $<1.5 \mathrm{~m}^{2}, 50 \mathrm{mg} ; \geqslant 1.5 \mathrm{~m}^{2}$, $60 \mathrm{mg}$ ) on days $1-14$ of a 21-day cycle.

This schedule was repeated until disease progression, development of unacceptable toxicity, or patient withdrawal of consent. If patients had a neutrophil count less than $1000 \mathrm{~mm}^{-3}$, platelet count less than $75 \times 10^{3} \mathrm{~mm}^{-3}$, serum creatinine more than $1.2 \mathrm{mg} \mathrm{dl}^{-1}$, infection with fever, or anorexia, diarrhoea, oral mucositis, or rash of grade 2 or higher, treatment with S-1 was suspended. In patients with febrile neutropaenia, grade 4 neutropaenia, grade 3-4 thrombocytopaenia, serum creatinine $>1.2 \mathrm{mg} \mathrm{dl}^{-1}$, or grade 3-4 diarrhoea, oral mucositis, or rash, doses of S-1 and cisplatin were reduced starting from the next cycle. In patients who had grade 3-4 vomiting or anorexia because of cisplatin, the dose of cisplatin was reduced. If heart failure or severe infusion reactions occurred, treatment with trastuzumab was discontinued.

Evaluations. The primary end point was RR. The secondary end points were overall survival (OS), progression-free survival (PFS), time to treatment failure (TTF), and adverse events. Tumours were assessed every 6 weeks until disease progression, and objective responses were evaluated according to the RECIST guidelines (version 1.1). For complete response (CR) or partial response (PR), confirmation 4 weeks after initial evaluation was necessary. An independent review committee assessed responses in all patients. OS was defined as the time from the date of enrollment to the date of death from any cause. PFS was defined as the time from the date of enrollment to the date of disease progression or death from any cause. TTF was defined as the time from the date of enrollment to the date when the treating physician decided to discontinue treatment for any reason. Physical examination and blood test were mandatory before each course, and left ventricular ejection fraction was assessed every 3 month during treatment. Adverse events were evaluated according to the National Cancer Institute Common Terminology Criteria for Adverse Events, version 4.0 .

Statistical analysis. The required sample size was estimated based on a threshold RR of $35 \%$ and an expected RR of $50 \%, 80 \%$ power, and an alpha value of 0.1 (one-sided) using the binomial test. Given $2 \%$ of ineligible patients, the target sample size was determined to be at least 50 patients. Efficacy was evaluated in all patients who received at least one dose of the study treatment. 
We used the Kaplan-Meier method to estimate survival curves and Greenwood's formula to calculate 95\% confidence intervals (CIs) for survival rates. Statistical analyses were conducted with R, version 3.0.1.

\section{RESULTS}

Patient background. Between July 2011 and May 2012, a total of 56 patients were enrolled from 29 hospitals in Japan. Two patients were ineligible because of inadequate renal function or the absence of measurable lesions. The characteristics of the 54 eligible patients are listed in Table 1 . The median age was 66 years (range $=34-75$ years). Two-thirds of patients had differentiated adenocarcinoma. Only three patients (6\%) had recurrent disease; the others had unresectable lesions. The most frequent sites of metastasis were the lymph nodes (81\%), followed by the liver (59\%). The proportions of IHC $3+$ and IHC $2+/$ FISH-positive tumours were $83 \%$ and $17 \%$, respectively.

\begin{tabular}{|c|c|}
\hline Characteristic & $n=54$ \\
\hline \multicolumn{2}{|l|}{ Age, years } \\
\hline $\begin{array}{l}\text { Median } \\
\text { Range }\end{array}$ & $\begin{array}{c}66 \\
34-75\end{array}$ \\
\hline \\
\hline $\begin{array}{l}\text { Male } \\
\text { Female }\end{array}$ & $\begin{array}{l}42(78 \%) \\
12(22 \%)\end{array}$ \\
\hline
\end{tabular}

Performance status

\begin{tabular}{|l|l}
\hline 0 & $42(78 \%)$
\end{tabular}

$12(22 \%)$

\section{Histological type}

\begin{tabular}{l|l}
\hline Differentiated & $36(67 \%)$ \\
Undifferentiated & $18(33 \%)$
\end{tabular}

Previous gastrectomy

\begin{tabular}{|l|r|}
\hline No & $45(83 \%)$ \\
\hline
\end{tabular}

Yes

$9(17 \%)$

\section{Unresectable/recurrent}

Unresectable

Recurrent with adjuvant chemotherapy

Recurrent without adjuvant chemotherapy

$51(94 \%)$

$2(4 \%)$

$1(2 \%)$

\section{Metastatic sites ${ }^{a}$}

\begin{tabular}{l|c} 
Lymph nodes & $44(81 \%)$ \\
Liver & $32(59 \%)$ \\
Lung & $5(9 \%)$ \\
Peritoneum & $5(9 \%)$ \\
Bone & $2(4 \%)$ \\
Other & $1(2 \%)$
\end{tabular}

HER2 status

$\mathrm{IHC} 3+$

IHC $2+/$ FISH positive

$45(83 \%)$

$9(17 \%)$

Abbreviations: $\mathrm{FISH}=$ fluorescence in situ hybridization; HER2 = human epidermal growth factor receptor type 2; $\mathrm{IHC}=$ immunohistochemistry.

${ }^{a}$ Some patients had multiple metastatic sites.
Efficacy. Of the 54 eligible patients, 1 patient did not receive any treatment per protocol because of a decrease in serum hemoglobin levels after study enrollment. Efficacy and safety analyses were therefore conducted in the full analysis set of the remaining 53 patients.

The median number of cycles was 6 (range $=1-27$ ), and the median relative dose intensity for $\mathrm{S}-1$, cisplatin, and trastuzumab was $76 \%, 83 \%$, and $96 \%$, respectively. At the time of analysis (August 2013), 51 patients had discontinued treatment. The main reason for discontinuation was progressive disease (31 patients), followed by adverse events (16 patients). Four patients underwent surgery because of a prominent response.

The confirmed RR based on RECIST (version 1.1) was $68 \%$ (95\% CI $=54-80 \% ; 80 \% \mathrm{CI}=58-76 \%$; Table 2 ), so the null hypothesis for the primary end point $(\mathrm{RR} \leqslant 35 \%)$ was rejected $(P<0.001)$. The confirmed RRs in the differentiated type cases $(n=35)$ and the undifferentiated type cases $(n=18)$ were $69 \%$ $(95 \% \mathrm{CI}=51-83 \%)$ and $67 \%(95 \% \mathrm{CI}=41-87 \%)$, respectively. Among 36 patients with CR or PR, the median time to response and duration of response were 41 days (range $=33-91$ days) and 208 days (range $=42-630$ days), respectively. The disease control rate, that is, the proportion of patients who had a CR, PR, or stable disease, was $94 \%$ (95\% CI $=84-99 \%)$. Two patients (4\%) had a CR. A waterfall plot of the confirmed best overall response for each patient is shown in Figure 1.

The median duration of follow-up at the time of analysis (August 2013) for the 53 patients was 13.5 months. The median OS was 16.0 months $(95 \% \mathrm{CI}=13.3$-not applicable), and the 1 -year OS rate was $67.9 \%$ (95\% CI $=56.5-81.7 \%$; Figure 2$)$. The median PFS was 7.8 months (95\% CI $=6.0-8.8$ months), and the 1 -year PFS rate was $17.0 \%$ (95\% CI $=9.4-30.8 \%$; Figure 2$)$. The median TTF was 5.7 months (95\% CI=4.2-7.1 months), and the 1 -year TTF rate was $5.1 \%(95 \% \mathrm{CI}=1.4-18.6 \%)$.

Safety. All adverse events that occurred in three or more patients are shown in Table 3. Among the haematological adverse events, the proportions of grade 3-4 neutropaenia and anaemia were $36 \%$ and $15 \%$, respectively. The most frequent common non-haematological toxicity was anorexia (any grade, 79\%; grade 3-4, 23\%). Except for anorexia, there were no grade 3 or 4 toxicities that occurred in more than $10 \%$ of patients. Creatinine was elevated in 24 of 53 patients (45\%). Grade 2 infusion-related reactions occurred in three patients $(6 \%)$. Heart failure did not occur in any patients.

There was one treatment-related death attributable to myelosuppression. This patient was judged as an ineligible case afterwards, because creatinine clearance before enrollment was $47.4 \mathrm{ml} \mathrm{min}^{-1}$. Furthermore, S-1 administration continued despite a serum creatinine level of $2.31 \mathrm{mg} \mathrm{dl}^{-1}$ on day 7. Renal dysfunction led to myelosuppression that progressed to death. Upon review of the patient's records, the data and safety

\begin{tabular}{|l|c|}
\hline \multicolumn{2}{|l|}{ Table 2. Objective response with confirmation based on RECIST } \\
\hline Total & $n=53$ \\
\hline Complete response & $2(4 \%)$ \\
\hline Partial response & $34(64 \%)$ \\
\hline Stable disease & $14(26 \%)$ \\
\hline Progressive disease & $3(6 \%)$ \\
\hline Response rate (95\% confidence interval) & $68 \%(54-80 \%)$ \\
\hline Disease control rate (95\% confidence interval) & $94 \%(84-99 \%)$ \\
\hline Abbreviation: RECIST = Response Evaluation Criteria in Solid Tumor. \\
\hline
\end{tabular}




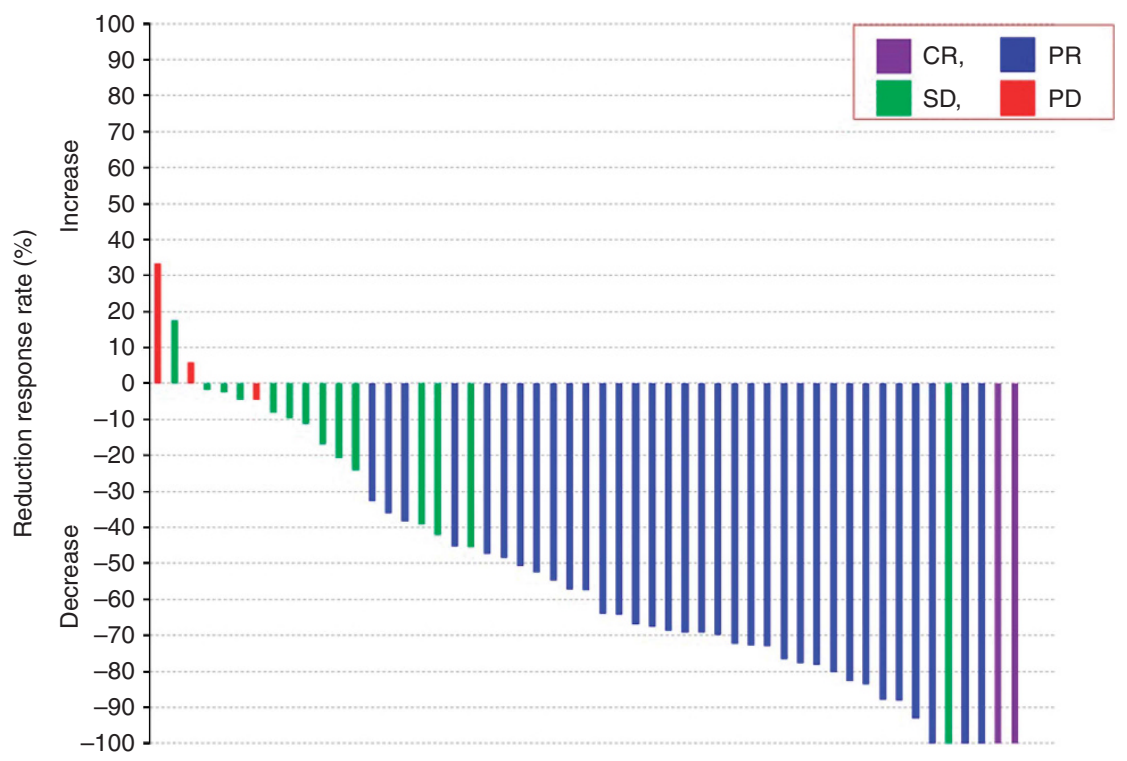

Figure 1. Waterfall plot of confirmed best overall response.
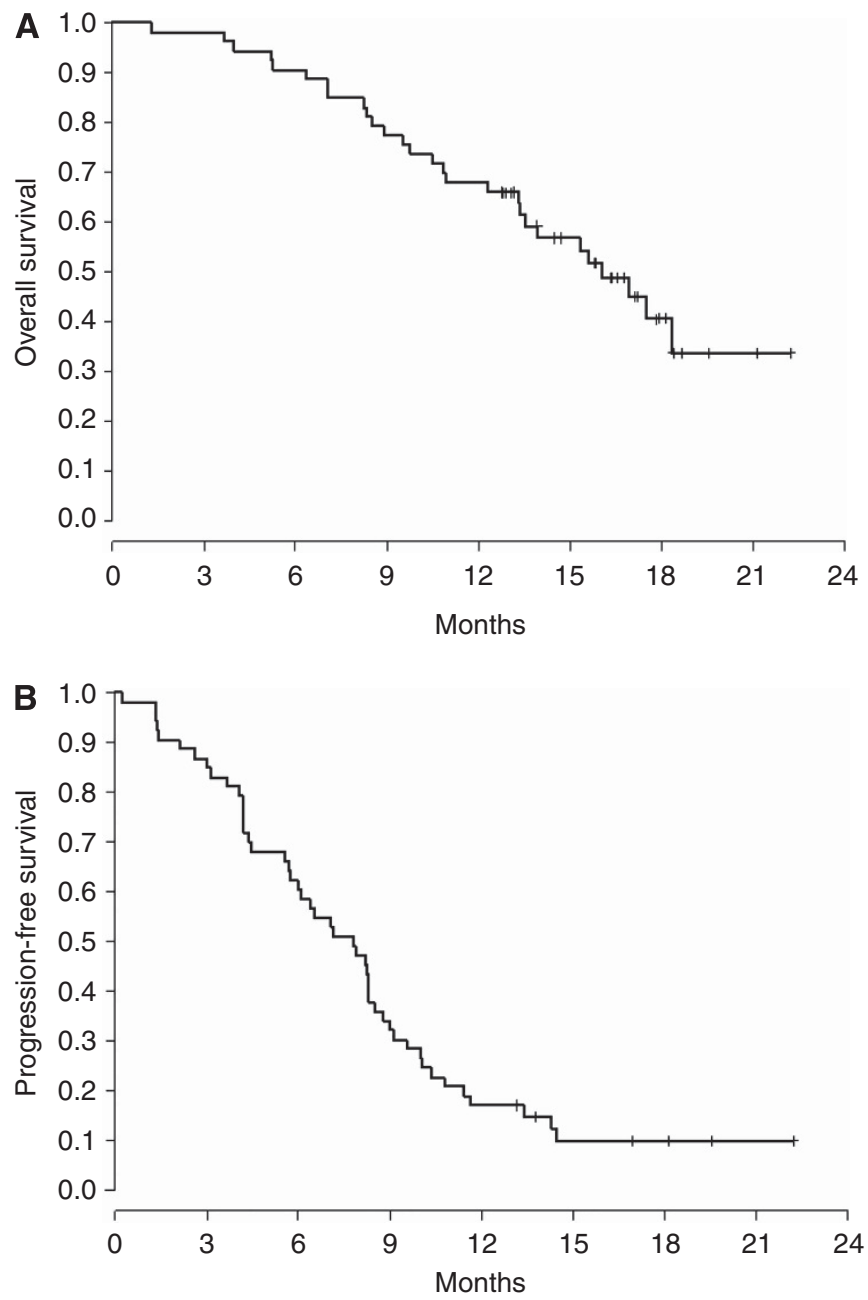

Figure 2. (A) The Kaplan-Meier overall survival and (B) progressionfree survival.

monitoring committee determined that the patient died from critical deviations from the eligibility criteria and treatment protocol.

\section{DISCUSSION}

This multicenter phase II study is the first clinical trial reporting the efficacy and safety of SP plus trastuzumab in patients with HER2-positive AGC. We obtained a much higher RR (68\%) than expected. The toxicity profile of our regimen was tolerable, and the incidence of grade 3-4 adverse events were similar to those of the SP regimen in the SPIRITS study (Koizumi et al, 2008). These results suggest that SP plus trastuzumab is a potential new treatment option for patients with HER2-positive AGC.

The ToGA study demonstrated that trastuzumab in combination with cisplatin plus capecitabine or fluorouracil was superior to cisplatin plus capecitabine or fluorouracil alone (Bang et al, 2010). The RR was $35 \%$ in the chemotherapy group and $47 \%$ in the trastuzumab plus chemotherapy group. In the aforementioned phase II study of a 3-week cycle of SP, the RR was $48 \%$, compared with $68 \%$ in the present study, suggesting that trastuzumab considerably enhanced the effectiveness of chemotherapy, which is consistent with the results of the ToGA study. In addition, the median OS and PFS in our study were 16.0 and 7.8 months, respectively, whereas the subgroup of Japanese patients in the trastuzumab arm of the ToGA study had a median OS and PFS of 15.9 and 6.2 months, respectively (Sawaki et al, 2012). Although these results must be interpreted with caution because of the differences between the ToGA study and our study in terms of patient characteristics, especially histologic type, the proportion of patients with HER2 IHC $3+$ tumours, and exclusion of patients with performance status $\geqslant 2$, trastuzumab may be a good addition to a S-1-based regimen. Experimental studies have reported that trastuzumab induces downregulation of thymidylate synthase expression. This mechanism has been implicated in the synergistic antitumour effect of S-1 plus trastuzumab against gastric cancer cell lines that overexpress HER2 (Tanizaki et al, 2010). Capecitabine and S-1 are both 5-FU derivatives, but were developed based on different concepts. Further studies of biomarkers and other predictors of outcomes are necessary to optimise the use of these drugs.

During the planning phase of this trial, a 5-week cycle of SP therapy was the mainstay of chemotherapy for AGC in Japan, based on the results of the SPIRITS study (Koizumi et al, 2008). As a molecular-targeted agent was combined with SP, the development of a 3-week cycle was planned. Results of phase II studies of a 3-week regimen of SP have been reported in gastric 


\begin{tabular}{|c|c|c|c|c|c|c|}
\hline & \multicolumn{4}{|c|}{ Grade } & \multirow[b]{2}{*}{$\begin{array}{l}\text { Any } \\
(\%)\end{array}$} & \multirow[b]{2}{*}{$\begin{array}{l}\text { Grade } \\
3-4(\%)\end{array}$} \\
\hline Event & 1 & 2 & 3 & 4 & & \\
\hline Leukopaenia & 17 & 18 & 3 & 1 & 74 & 8 \\
\hline Neutropaenia & 8 & 5 & 14 & 5 & 60 & 36 \\
\hline Febrile neutropaenia & 0 & 0 & 1 & 1 & 4 & 4 \\
\hline Anaemia & 5 & 22 & 6 & 2 & 66 & 15 \\
\hline Thrombocytopaenia & 20 & 6 & 0 & 0 & 49 & 0 \\
\hline Anorexia & 15 & 15 & 12 & 0 & 79 & 23 \\
\hline Fatigue & 18 & 14 & 2 & 0 & 64 & 4 \\
\hline Nausea & 20 & 12 & 1 & 0 & 62 & 2 \\
\hline Hypoalbuminaemia & 14 & 6 & 5 & 0 & 47 & 9 \\
\hline Hypertension & 9 & 12 & 1 & 0 & 42 & 2 \\
\hline Creatinine increased & 21 & 0 & 3 & 0 & 45 & 6 \\
\hline Diarrhoea & 10 & 7 & 4 & 0 & 40 & 8 \\
\hline Oral mucositis & 10 & 6 & 1 & 0 & 32 & 2 \\
\hline Skin rash & 12 & 1 & 0 & 0 & 25 & 0 \\
\hline Vomiting & 7 & 3 & 3 & 0 & 25 & 6 \\
\hline ALT increased & 11 & 2 & 0 & 0 & 25 & 0 \\
\hline Constipation & 7 & 4 & 0 & 0 & 21 & 0 \\
\hline Dysgeusia & 7 & 3 & 0 & 0 & 19 & 0 \\
\hline AST increased & 9 & 0 & 0 & 0 & 17 & 0 \\
\hline Blood bilirubin increased & 6 & 2 & 0 & 0 & 15 & 0 \\
\hline Edema & 6 & 2 & 0 & 0 & 15 & 0 \\
\hline $\begin{array}{l}\text { Peripheral sensory } \\
\text { neuropathy }\end{array}$ & 1 & 5 & 0 & 0 & 11 & 0 \\
\hline Epistaxis & 3 & 1 & 0 & 0 & 8 & 0 \\
\hline Hiccups & 4 & 0 & 0 & 0 & 8 & 0 \\
\hline Fever & 2 & 2 & 0 & 0 & 8 & 0 \\
\hline Infusion-related reaction & 0 & 3 & 0 & 0 & 6 & 0 \\
\hline Alopecia & 2 & 1 & 0 & 0 & 6 & 0 \\
\hline Abdominal pain & 1 & 2 & 0 & 0 & 6 & 0 \\
\hline Skin hyperpigmentation & 2 & 1 & 0 & 0 & 6 & 0 \\
\hline
\end{tabular}

cancer and lung cancer (Lee et al, 2008; Choi et al, 2010; Kubota et al, 2010). Recently, a phase III trial comparing the standard 5 -week cycle of SP with a 3-week cycle of SP was conducted in patients with AGC. This trial showed that the median PFSs in the 3-week and 5-week cycle groups were 5.5 and 4.9 months, respectively, and it concluded that a 3-week cycle of SP was superior to a 5 -week cycle of SP in terms of PFS $(P=0.042)$ (Ryu et al, 2013). We therefore expected that a 3-week regimen of SP plus trastuzumab would be more effective than a 5-week regimen of SP plus trastuzumab. Although the dose intensity of cisplatin (20 $\mathrm{mg} \mathrm{m}^{-2}$ per week) in a 3-week SP regimen was $25 \%$ lower than that (26.7 $\mathrm{mg} \mathrm{m}^{-2}$ per week) in the ToGA study regimen, the RR (48\%) of 3-week SP regimen was higher than that $(35 \%)$ of the ToGA regimen. Thus, we considered that the dose $\left(60 \mathrm{mg} \mathrm{m}^{-2}\right)$ of cisplatin was adequate in this 3 -week SP regimen.

In this study, we limited subjects to patients with measurable lesions assessable according to RECIST guidelines (version 1.1). In clinical practice, however, many patients with gastric cancer have no measurable lesions, such as those with peritoneal metastasis. We are therefore conducting another phase II study in patients who have HER2-positive AGC without measurable lesions (HERBIS-1B; UMIN000007941) to confirm the usefulness of this regimen in this subgroup.

In conclusion, although this was not a randomised controlled study, our results suggest that SP plus trastuzumab has a good toxicity profile and promising efficacy, justifying the further study of regimens that contain SP and trastuzumab.

\section{ACKNOWLEDGEMENTS}

This work was supported by Taiho Pharmaceutical. This study was conducted as a collaborative effort of the Osaka Gastrointestinal Cancer Chemotherapy Study Group (OGSG), Hokkaido Gastrointestinal Cancer Study Group (HGCSG), and Tohoku Clinical Oncology Research and Education Society (T-CORE). We are indebted to the last Professor Hiroya Takiuchi, who played a pivotal role in this study. We thank Y Sato and H Hagimoto who provided editorial support on behalf of Taiho Pharmaceutical.

\section{CONFLICT OF INTEREST}

Y Kurokawa, H Imamura, Y Komatsu, Y Doki, and T Tsujinaka received speaker honoraria from Taiho Pharmaceutical. Y Komatsu and Y Doki received unrestricted research grant from Taiho Pharmaceutical. The remaining authors declare no conflict of interest.

\section{REFERENCES}

Bang YJ, van Cutsem E, Feyereislova A, Chung HC, Shen L, Sawaki A, Lordick F, Ohtsu A, Omuro Y, Satoh T, Aprile G, Kulikov E, Hill J, Lehle M, Ruschoff J, Kang YK (2010) Trastuzumab in combination with chemotherapy versus chemotherapy alone for treatment of HER2-positive advanced gastric or gastro-oesophageal junction cancer (ToGA): a phase 3 , open-label, randomised controlled trial. Lancet 376: 687-697.

Choi IS, Lee KW, Kim KH, Kim YJ, Kim JH, Lee JS (2010) Three-weekly S-1 plus cisplatin chemotherapy as first-line treatment for advanced gastric cancer. Med Oncol 27: 992-997.

Cunningham D, Starling N, Rao S, Iveson T, Nicolson M, Coxon F, Middleton G, Daniel F, Oates J, Norman AR (2008) Capecitabine and oxaliplatin for advanced esophagogastric cancer. N Engl J Med 358: 36-46.

Diasio RB (1999) Clinical implications of dihydropyrimidine dehydrogenase inhibition. Oncology (Williston Park) 13: 17-21.

Eisenhauer EA, Therasse P, Bogaerts J, Schwartz LH, Sargent D, Ford R, Dancey J, Arbuck S, Gwyther S, Mooney M, Rubinstein L, Shankar L, Dodd L, Kaplan R, Lacombe D, Verweij J (2009) New response evaluation criteria in solid tumours: revised RECIST guideline (version 1.1). Eur J Cancer 45: 228-247.

Ferlay J, Shin HR, Bray F, Forman D, Mathers C, Parkin DM (2010) Estimates of worldwide burden of cancer in 2008: GLOBOCAN 2008. Int J Cancer 127: 2893-2917.

Hofmann M, Stoss O, Shi D, Büttner R, van de Vijver M, Kim W, Ochiai A, Rüschoff J, Henkel T (2008) Assessment of a HER2 scoring system for gastric cancer: results from a validation study. Histopathology 52: 797-805.

Hudis CA (2007) Trastuzumab-mechanism of action and use in clinical practice. $N$ Engl J Med 357: 39-51.

Japanese Gastric Cancer Association (2011) Japanese gastric cancer treatment guidelines 2010 (ver. 3). Gastric Cancer 14: 113-123.

Kang YK, Kang WK, Shin DB, Chen J, Xiong J, Wang J, Lichinitser M, Guan Z, Khasanov R, Zheng L, Philco-Salas M, Suarez T, Santamaria J, Forster G, Mccloud PI (2009) Capecitabine/cisplatin versus 5-fluorouracil/ cisplatin as first-line therapy in patients with advanced gastric cancer: a randomised phase III noninferiority trial. Ann Oncol 20: 666-673. 
Koizumi W, Kurihara M, Nakano S, Hasegawa K (2000) Phase II study of S-1, a novel oral derivative of 5-fluorouracil, in advanced gastric cancer. For the S-1 Cooperative Gastric Cancer Study Group. Oncology 58: 191-197. Koizumi W, Narahara H, Hara T, Takagane A, Akiya T, Takagi M,

Miyashita K, Nishizaki T, Kobayashi O, Takiyama W, Toh Y, Nagaie T, Takagi S, Yamamura Y, Yanaoka K, Orita H, Takeuchi M (2008) S-1 plus cisplatin versus S-1 alone for first-line treatment of advanced gastric cancer (SPIRITS trial): a phase III trial. Lancet Oncol 9: 215-221.

Kubota K, Sakai H, Yamamoto N, Kunitoh H, Nakagawa K, Takeda K, Ichinose Y, Saijo N, Ariyoshi Y, Fukuoka M (2010) A multi-institution phase I/II trial of triweekly regimen with S-1 plus cisplatin in patients with advanced non-small cell lung cancer. $J$ Thorac Oncol 5: 702-706.

Lee JL, Kang HJ, Kang YK, Ryu MH, Chang HM, Kim TW, Sohn HJ, Kim H, Lee JS (2008) Phase I/II study of 3-week combination of S-1 and cisplatin chemotherapy for metastatic or recurrent gastric cancer. Cancer Chemother Pharmacol 61: 837-845.

Macdonald JS, Smalley SR, Benedetti J, Hundahl SA, Estes NC, Stemmermann GN, Haller DG, Ajani JA, Gunderson LL, Jessup JM, Martenson JA (2001) Chemoradiotherapy after surgery compared with surgery alone for adenocarcinoma of the stomach or gastroesophageal junction. N Engl J Med 345: 725-730.

Ryu MH, Baba E, Lee KH, Boku N, Park YI, Hyodo I, Nam BH, Esaki T, Ryoo BY, Song EK, Cho S, Lee SS, Kang WK, Yang SH, Zang DY, Shin DB, Park SR, Shinozaki K, Takano T, Kang Y-K (2013) Phase III trial of a 3-weekly versus 5 -weekly schedule of S-1 plus cisplatin (SP) combination chemotherapy for first-line treatment of advanced gastric cancer (AGC): SOS study. J Clin Oncol (suppl) 31: abstr LBA4024.
Sakata Y, Ohtsu A, Horikoshi N, Sugimachi K, Mitachi Y, Taguchi T (1998) Late phase II study of novel oral fluoropyrimidine anticancer drug S-1 (1 M tegafur-0.4 M gimestat-1 $\mathrm{M}$ otastat potassium) in advanced gastric cancer patients. Eur J Cancer 34: 1715-1720.

Sawaki A, Ohashi Y, Omuro Y, Satoh T, Hamamoto Y, Boku N, Miyata Y, Takiuchi H, Yamaguchi K, Sasaki Y, Nishina T, Satoh A, Baba E, Tamura T, Abe T, Hatake K, Ohtsu A (2012) Efficacy of trastuzumab in Japanese patients with HER2-positive advanced gastric or gastroesophageal junction cancer: a subgroup analysis of the Trastuzumab for Gastric Cancer (ToGA) study. Gastric Cancer 15: 313-322.

Shirasaka T, Shimamato Y, Ohshimo H, Yamaguchi M, Kato T, Yonekura K, Fukushima M (1996) Development of a novel form of an oral 5-fluorouracil derivative (S-1) directed to the potentiation of the tumor selective cytotoxicity of 5-fluorouracil by two biochemical modulators. Anticancer Drugs 7: 548-557.

Tanizaki J, Okamoto I, Takezawa K, Tsukioka S, Uchida J, Kiniwa M, Fukuoka M, Nakagawa K (2010) Synergistic antitumor effect of S-1 and HER2-targeting agents in gastric cancer with HER2 amplification. Mol Cancer Ther 9: 1198-1207.

This work is published under the standard license to publish agreement. After 12 months the work will become freely available and the license terms will switch to a Creative Commons AttributionNonCommercial-Share Alike 3.0 Unported License. 